Meta

Journal des traducteurs

Translators' Journal

\title{
Modèle unifié d'exécution de prestations de traduction/traducteur avec applications à la pratique professionnelle et à la formation des traducteurs
}

\section{Daniel Gouadec}

Volume 50, numéro 4, décembre 2005

Pour une traductologie proactive - Actes

For a Proactive Translatology — Proceedings

Por una traductología proactiva - Actas

URI : https://id.erudit.org/iderudit/019844ar

DOI : https://doi.org/10.7202/019844ar

Aller au sommaire du numéro

Éditeur(s)

Les Presses de l'Université de Montréal

ISSN

0026-0452 (imprimé)

1492-1421 (numérique)

Découvrir la revue

Citer cet article

Gouadec, D. (2005). Modèle unifié d'exécution de prestations de traduction/traducteur avec applications à la pratique professionnelle et à la formation des traducteurs. Meta, 50(4). https://doi.org/10.7202/019844ar
Résumé de l'article

Présentation d'un modèle générique d'exécution de la prestation du traducteur [traducteurlocaliseur-sous-titreur-adaptateur] en fonction du cycle de vie du matériau traité [texte, graphisme, vidéo, site Web, etc.] L’architecture, modulaire, combine des opérations en modules, des modules en blocs de modules, des blocs de modules en activités et des activités en éléments de prestation. Le modèle propose une analyse en chemin critique des interventions croisées des diverses 'fonctions' mobilisées dans, et par, l'exécution de la prestation. L'extraction d'opérations unitaires (atomiques) permet de repérer l'apport de chaque variable et l'effet de chaque variation en échappant aux interférences externes aux opérations elles-mêmes.

L'article propose de nouvelles orientations de recherche en fonction des apports du modèle. Il indique en quoi le modèle peut servir à l'optimisation de la prestation du traducteur. Il propose une application du modèle à l'organisation de la formation des traducteurs. 


\title{
Modèle unifié d'exécution de prestations de traduction/traducteur avec applications à la pratique professionnelle et à la formation des traducteurs
}

\author{
DANIEL GOUADEC \\ Université de Haute Bretagne, Rennes, France \\ daniel.gouadec@uhb.fr
}

\begin{abstract}
RÉSUMÉ
Présentation d'un modèle générique d'exécution de la prestation du traducteur [traducteurlocaliseur-sous-titreur-adaptateur] en fonction du cycle de vie du matériau traité [texte, graphisme, vidéo, site Web, etc.] L'architecture, modulaire, combine des opérations en modules, des modules en blocs de modules, des blocs de modules en activités et des activités en éléments de prestation. Le modèle propose une analyse en chemin critique des interventions croisées des diverses 'fonctions' mobilisées dans, et par, l'exécution de la prestation. L'extraction d'opérations unitaires (atomiques) permet de repérer l'apport de chaque variable et l'effet de chaque variation en échappant aux interférences externes aux opérations elles-mêmes.

L'article propose de nouvelles orientations de recherche en fonction des apports du modèle. Il indique en quoi le modèle peut servir à l'optimisation de la prestation du traducteur. Il propose une application du modèle à l'organisation de la formation des traducteurs.
\end{abstract}

\begin{abstract}
This article purports to provide a 'universal' model of the conditions and workings of translation service provision, taking into account the variety of types of materials and types of functions that are, or may be, involved. The model is built from 'operations' that combine to build up modules and module sets which, in turn, make up thet activities that are organised into elements further sequenced into the overall service provision. It offers a critical path analysis of the translation cycle, with due consideration to the various operators and functions that do influence the processes or interact in the course of the processings. Working with recombinant unitary operations makes it possible to pinpoint the influence of each and every variable and to clearly see the effects of variation without inteference from influences outside the operations themselves.

The article mentions new ways for research and study along those lines, explains how the model may be put to advantage to optimise the translator's work process, and describes how it may impact the training of translators positively.
\end{abstract}

\section{MOTS-CLÉS/KEYWORDS}

prestation du traducteur, modèle unifié, application professionnelle, application pédagogique

Le modèle présenté ici est un modèle d'exécution de prestations de traduction : il modélise l'activité professionnelle standard de la personne sociale que l'on continue d'appeler « traducteur ». Il est centré sur l'ensemble des procédures qui conduisent à la production d'un matériau traduit autonome ou intégré à un support et/ou à un instrument et non sur le processus de la traduction-transfert en tant que tel. 


\section{GENESE}

Le modèle repose sur les résultats de quatre analyses.

1. Une première analyse effectuée en 1984 afin de déterminer les conditions optimales de mise en place, en urgence, d'une organisation permettant de gérer, en contexte d'apprentissage/formation, la traduction collective, par des étudiants de maîtrise en traduction, d'un mode d'emploi de logiciel comptant plusieurs centaines de pages.

2. Une deuxième analyse effectuée en 1985 dans le but de transposer le modèle d'exécution de traductions ${ }^{1}$ ainsi élaboré au contexte pédagogique auquel il apportait notamment la responsabilisation des étudiants, l'encadrement des pratiques, et, surtout, des procédures de mises en commun et de corrections par étages telles que les interventions du formateur s'en trouvaient considérablement simplifiées ${ }^{2}$.

3. Une troisième analyse, dite d'employabilité, reposant sur la décomposition des contenus de prestations de traduction/traducteurs pour parvenir à la définition claire et à l'optimisation de profils de compétences ${ }^{3}$.

4. Une quatrième analyse, conduite au titre d'un projet MLIS (1997-1999), visant la mise en place d'une organisation rationnelle d'exécution de prestations de traduction, en environnement industriel, en prenant en compte toutes les variables, dans toutes les situations de métiers (traduction, localisation, sous-titrage, etc.)

Ces études confirment que toute prestation « de traduction » ou « avec traduction » fait référence à un matériau existant défini selon toute combinaison des principales variables suivantes : nature, type, support, mode, moyens, outils mis en œuvre, destinations, autonomie ou intégration et, dans ce dernier cas, nature de l'intégrateur (livre, jeu vidéo, site Web, film, etc.) Les processus de transformation appliqués à ce matériau existant aboutissent à un matériau dérivé luimême défini selon toute combinaison des mêmes variables que précédemment, à savoir : nature, type, support, mode, moyens, destinations, outils mis en œuvre, autonomie ou intégration et, dans ce dernier cas, nature de l'intégrateur (livre, jeu vidéo, site Web, film, etc.) Le traitement, qui est largement déterminé par la nature de l'instrument dans lequel s'intègre le matériau traduit, s'effectue selon un ensemble d'opérations-processus liés ou chaînés, dont les caractéristiques sont déterminées par des conventions, des spécifications (génériques et spécifiques), des règles de conformité corrigées des écarts culturels/linguistiques/communicatifs types, mais aussi par les outils, moyens et ressources mis en œuvre, par les opérateurs impliqués, et par les séquencements opérationnels.

Sur l'autre versant, le modèle considère toute prestation de traducteur ou avec traducteur comme un ensemble d'activités séquencées dont chacune (a) mobilise un faisceau de compétences réputées optimisables, (b) sollicite des comportements (optimisables, par définition, dans tout modèle) et (c) appelle des stratégies dont chacune concerne au moins une procédure applicable à un matériau donné ou à une composante particulière d'un matériau donné pour atteindre un objectif défini et qualifié.

\section{COMPOSANTES}

En fait, le modèle «traduit », au niveau le plus large, un cycle de vie d'un matériau/instrument ${ }^{4}$ séquencé en : conception d'un matériau $\Rightarrow$ production/fabrication de ce matériau $\Rightarrow$ décision de faire traduire $\Rightarrow$ désassemblage $\Rightarrow$ transformation $^{5} \Rightarrow$ réassemblage $\Rightarrow$ intégration ou réintégration $\Rightarrow$ diffusion. 
Ce cycle de vie générique se décompose activités ou tâches, elles-mêmes décomposables en sous-activités ou sous-tâches, et ainsi de suite, jusqu'à ce que l'on atteigne le stade de l'opération que l'on dira élémentaire.

L’idée qui sous-tend l'ensemble de la modélisation est que la décomposition de la prestation de traduction/traducteur fait apparaître plusieurs niveaux cohérents :

- l'élément de prestation dans le cadre de la prestation globale [exemples : traitement d'un lot ou production d'un composant spécifique, création de la mémoire de traduction, relecture],

- l'activité [exemple : élucidation, validation de la terminologie, relecture ${ }^{6}$ ],

- l'ensemble cohérent d'opérations ou module [exemple : préparation de la version à traduire ou retraduire effectivement],

- l'opération « atomique » ou opération élémentaire à objet singulier et processus unique relevant

donc, probablement, d'une procédure unique.

La construction du modèle doit prendre en compte les quatre niveaux simultanément, par emboîtement ascendant ou descendant selon les perspectives.

L'architecture de base est la séquence des activités - qui aboutit généralement à l'exécution d'un élément de prestation au moins. Aux niveaux suivants, l'analyse montre que (1) chaque activité est elle-même décomposable en une séquence de «blocs d'opérations » ou modules et que (2) chaque module est lui-même décomposable en opérations.

Chaque séquence [séquence d'activités, enchaînement ou «bloc » de modules, suite d'opérations] est décrit comme un enchaînement dans lequel une activité/un module/une opération dont le résultat alimente une activité/un module/une opération subséquente(e) ou ultérieur(e) être effectué(e) [1] en temps voulu ${ }^{7}$ et [2] dans des conditions de qualité requises pour un déroulement optimal de l'activité/du module/de l'opération alimenté(e). On peut ainsi parler d'organisation en chemin critique de l'exécution de la prestation et, dans ce cadre générique, de l'exécution des activités, modules ou opérations, puisque chacun des niveaux, correspond à une organisation avec prérequis, conditions nécessaires, emboîtements et marges, et puisque chaque élément comme l'ensemble comporte toujours une dimension économique puisque l'exécution de prestations de traductions intervient toujours en situation de ressources finies [temps, budget, ressources humaines, matériels et logiciels, outils, etc.]

Le chemin critique générique universellement applicable aux prestations de traduction est évoqué dans un récent article du numéro anniversaire de META $^{8}$. Les applications correspondant aux diverses combinaisons d'opérateurs sont présentés à l'adresse www.qualitrad.net sur un site consacré, plus généralement, à la qualité en traduction professionnelle. Le parcours d'un traducteur donné exécutant une prestation donnée fait appel à tel ou tel constituant du modèle en fonction des conditions d'objet + objectif + processus (et donc de procédure) applicables à un moment donné de l'exécution de sa prestation, selon les conditions optimales de séquencement caractérisant le chemin critique particulier applicable. Le parcours générique est donc la somme de tous les parcours particuliers possibles, une opération donnée étant présente ou absente selon les cas et, lorsqu'elle est présente, susceptible de variabilité.

Le modèle prend en compte toutes les opérations qui apparaissent, à un titre quelconque, dans le processus d'exécution de prestations de traduction. Il peut s'agir aussi bien, par exemple, du calibrage d'un élément de matériau donné que de l'élucidation d'une valeur conceptuelle ou du transfert d'une valeur conceptuelle ou du contrôle de la qualité du transfert d'une phrase ou d'un contrôle de qualité fonctionnelle ou du remplacement d'un concordant terminologique par une nouvelle forme homologuée par le donneur d'ouvrage et ainsi de suite.

Selon le modèle, une opération donnée ${ }^{9}$ :

- doit intervenir en un temps T optimisé, qu'il appartient au modèle de définir dans une séquence elle-même optimisée (en fonction du chemin critique générique ou spécifique applicable) 
- doit se dérouler selon des spécifications générales - celles qui s'appliquent à toutes les opérations de même nature dans toutes les prestations de traduction - et des spécifications particulières - celles qui s'appliquent spécifiquement dans le cadre de la prestation considérée en vertu des exigences, requêtes, recommandations, etc. de tel ou tel opérateur autorisé, qu'il appartient également au modèle de catégoriser.

- est éventuellement soumise à des normes ou à des standards, selon les conditions définies.

- s'effectue, dans les cas extrêmes - identifiés par le modèle - selon des contraintes de gabarits ou modèles à préciser.

- porte, dans les conditions ainsi fixées, sur un matériau donné (dont la nature et les autres caractéristiques sont nécessairement identifiées et optimisées) auquel s'appliquent nécessairement des prérequis structurés, identifiables, et analysables. Ce matériau constitue le matériau principal en entrée du processus. Il n'est admis en entrée du processus que si tous les prérequis sont remplis.

Les prérequis concernant un matériau donné sont le résultat de l'application d'une série de traitements organisée en séquence selon le schéma ci-après :

1. Sélection

2. Extraction

3. Paramétrage

4. Mise en place dans l'environnement de traitement

5. Tests et essais

6. Validation et qualification

7. Intégration à l'environnement de travail

Chacun de ces traitements présuppose la mise en place du filtre de la combinaison voulue de conditions et spécifications dont certaines sont d'ordre générique et d'autres d'ordre particulier. Le modèle définit et analyse ces dernières en ayant pour objectif de prévenir les défaillances du processus de transformation.

- peut mettre en œuvre un matériau primaire (matériau préexistant) et, généralement, de la matière première. Pour le processus de transfert, par exemple, la matière première inclut les savoirs requis, les filtres contextuels, des composants linguistiques (terminologie, phraséologie, modèles stéréotypiques) des modèles d'organisation, et ainsi de suite.

Le matériau primaire et la matière première ne sont en principe admis dans le processus d'exécution de l'opération qu'à la condition que les mêmes prérequis que précédemment (prérequis applicables au matériau principal) soient garantis.

- met en œuvre, au moment voulu de la séquence, un ou plusieurs opérateurs et un ou plusieurs outils. Opérateur(s) et outil(s)/instrument(s) sont eux-mêmes soumis à la chaîne de prérequis concaténant sélection, extraction, paramétrage, mise en place, tests et essais, validation et qualification, intégration à l'environnement de travail. Dans le cas des opérateurs comme des outils, la sélection, par exemple, fait appel à des critères de compétence, performance, coût, etc. car, une fois encore, chaque traitement de la chaîne est filtré par la combinaison voulue de conditions et de spécifications additionnelles - également définies et analysées dans le modèle. - intervient, en tant qu'opération-processus, lorsque toutes les conditions du modèle sont réunies de telle sorte qu'elle se déroule dans des conditions optimales et produit un résultat lui-même optimal. A moins qu'il ne constitue lui-même le matériau sur lequel porte la (ou une) opération subséquente - avec son enchaînement de prérequis - le matériau obtenu en sortie de l'opération-processus est soumis à validation (au sens large).

Le modèle identifie et construit chacune des opérations élémentaires selon le schéma cidessus et chaque « atome » ainsi constitué fait l'objet de tests systématiques pour validation en situation de formation, puis en situation professionnelle - la différence entre l'une et l'autre résidant 
principalement dans les degrés de contrainte temporelle et financière applicables puisque les conditions de formation considérées sont une émulation pure et simple du modèle professionnel au sens où la formation est rigoureusement calquée, en tout point et en toute chose, à l'exception des composantes financières et économiques, sur ce modèle.

La technique utilisée est celle du traçage par enregistrement/consignation du matériau en entrée et du matériau en sortie ainsi que des évolutions du matériau en cours de processus. En clair, pour chaque opération identifiée sur la base d'une combinaison d'objet à traiter [objet dont la mise en place respecte tous les prérequis exigibles] et d'objectif à atteindre [objectif exprimé en une combinaison exhaustive de résultat escompté et de spécifications à respecter ${ }^{10}$, dont chacune constitue une variable de filtre] :

a) le matériau en entrée ainsi que tout matériau primaire disponible et toute matière première requise sont définis, mis en place, qualifiés et consignés ;

b) les évolutions de l'opération sont suivies par le biais des éventuelles verbalisations de l'opérateur ou des opérateurs (en cas de travail de groupe, qui favorise la verbalisation et les commentaires et justifications) et des évolutions du matériau traité à mesure que le processus avance ;

c) le matériau en sortie est également consigné, puisque chaque opérateur l'enregistre directement ou indirectement.

\section{INTERET DU MODELE}

L'intérêt d'une modélisation fondée sur la définition d'opérations élémentaires systématiquement inscrites dans l'architecture générale d'exécution de prestations de traduction réside d'abord dans le fait qu'elle se prête à l'expérimentation et à la validation. Travailler sur les opérations élémentaires permet, entre autres, de :

- multiplier les points ou moments, qui, lors de l'exécution des prestations, sont susceptibles de constituer autant de jalons au fil des chemins critiques ;

- délimiter finement les « objets » de processus et donc de procédures en partant des éléments les plus petits sans pour autant les couper de leurs intégrateurs, puisque les systèmes de conditions et spécifications, d'une part, et les emboîtements de prérequis, d'autre part, garantissent la cohérence globale ;

- prendre en compte tout élément qui apparaît, ou devient, significatif et construire la partie du modèle s'y rapportant sans pour autant remettre radicalement en cause l'existant ; - faire jouer toutes les combinaisons d'éléments envisageables dans la création de modules, puis dans l'agencement de ces modules entre eux ;

- repérer et identifier toutes les variables possibles, les traiter une à une, et les faire permuter afin de déterminer l'impact des variations sur l'organisation de l'exécution de la prestation au niveau de l'opération considérée, puis du module, puis de l'ensemble du chemin critique ; - repérer et identifier, pour chaque combinaison d'opération + processus + procédure élémentaire, les contributions sectorielles en termes de spécifications-conditions et en termes de prérequis.

La définition et l'optimisation des opérations élémentaires permettent de déterminer les apports et contributions souhaitables ou nécessaires des disciplines connexes en termes de savoirs, compétences, savoir-faire et savoir être qui interviennent ou sont susceptibles d'intervenir pour chacune d'entre elles.

Les opérations élémentaires étant analysées puis optimisées une à une, elles sont recombinées : 
a) en séries, lorsque la même opération élémentaire se répète [l'objectif étant de déterminer les variables significatives et les variations induites par la concaténation] et/ou

b) en modules, lorsque des opérations ou des séries d'opérations se combinent en une séquence d'opérations correspondant à une combinaison d'objet + objectif + spécifications de plus haut niveau. Les modules sont à leur tour agencés et optimisés en activités puis en éléments (ou composantes) de prestation et les éléments ou composantes de prestation sont à leur tour réorganisés en prestation selon les chemins critiques précédemment évoqués.

À chacun des niveaux, l'expérimentation et l'analyse d'optimisation visent le repérage et l'élimination des causes d'éventuelles défaillances de processus induisant des défauts de qualité du matériau issu de l'opération ou de l'activité ou de l'élément de prestation et, à terme, du matériau traduit. Elles permettent ainsi d'éliminer tout dysfonctionnements de l'instrument intégrant le matériau traduit et son support. L'analyse du processus porte sur les variables d'adaptation des ressources, instruments et moyens aux spécifications. Toute défaillance est corrigée par reprise du dispositif applicable à l'opération ou au module ou à l'activité ou à l'élément de prestation à partir d'une nouvelle série de combinaisons d'objets + objectifs + spécifications, en introduisant ou en faisant permuter toute variable pertinente, jusqu'à ce que le processus soit réputé infaillible ou, au moins, que toute cause rédhibitoire soit éliminée.

L'identification précise et rigoureuse d'opérations élémentaires, de modules, et d'activités conduit à définir ce que l'on appellera des profils fonctionnels adaptés qui sont en fait des profils de compétences [combinant savoirs, savoir faire ou habilités stratégiques, et savoir être] par opération, par module [groupe d'opérations liées] et par activité [bloc de modules solidaires]. La référence initiale aux profils de compétences d'opérateurs ${ }^{11}$ a vite été abandonnée en raison de la relative confusion des étiquetages professionnels et remplacée par des profils de compétences par opérations (opérations qui peuvent être distribuées diversement entre les opérateurs).

Le modèle établit donc des faisceaux de compétences par opération, par module et par activité et donc des « compétences par fonctions » puisque les fonctions se rattachent aux activités et/ou aux éléments de prestation. Il reste alors simplement, soit à répartir les fonctions entre les opérateurs disponibles pour une prestation donnée, soit à créer des profils d'opérateurs (et donc des profils de compétences) à fonctions ou combinaisons de fonctions spécifiques. Dans le premier cas de figure, on se situe dans une logique de gestion; dans le second, on se situe dans une logique de formation. Dans un cas comme dans l'autre, on raisonne en termes de «profils de métiers » et parfois même de «profils d'environnement ».

Les fonctions considérées sont représentées en annexe 2. Elles incluent (côté traducteur) :

- la gestion de projet, subdivisée en une fonction commerciale + financière + comptable + gestionnaire (incluant, le cas échéant, une fonction de pilotage d'exécution de prestations de traduction),

- la préparation, subdivisée en trois volets :

- préparation de l'environnement (essentiellement logistique)

- préparation du matériau elle-même décomposable en désassemblage, préparation du kit de traduction, mise en place de la version à traduire ou retraduire effectivement et, le cas échéant, de la version d'appui

- préparation de la matière première avec les sous-fonctions de documentation-recherche, terminologie, phraséologie et création-traitement des mémoires de traduction

- les transferts,

- les contrôles de qualité, avec les éventuels volets de relecture, révision, validation, retraduction et, pour chacun d'entre eux, application des profils à un ou plusieurs aspects de la qualité (qualité 
linguistique-stylistique-rédactiopnnelle, qualité technique-factuelle-sémantique, qualité des transferts, qualité fonctionnelle, ergonomie, etc.)

- l'intégration de premier niveau, ou intégration du matériau traduit sur son support de diffusion ou support fonctionnel,

- l'intégration de second niveau, ou intégration de matériau traduit et de son support dans

l'instrument final,

- les tests.

Ces fonctions renvoient diversement à la notion de métiers de la traduction qui remplace avantageusement celle de traduction et s'ouvrent sur une diversité d'applications par domaines, outils, technologies, supports et types de matériaux. Elles permettent en même temps de réintégrer l'ensemble des métiers sous le chapeau du cœur de métier que reste la traduction par-delà cette diversité d'applications.

\section{APPLiCATION PROFESSIONNELLE}

Le modèle propose un processus d'exécution de prestations qui optimise les déterminants et les variables par opération en intégrant la dimension économique. Ce modèle intègre les variables génériques ci-après :

- Nature et état du matériau en entrée

[application de l'ensemble de prérequis]

- Finalité de traitement exprimé en résultat escompté ou requis

[application de prérequis en termes de spécifications et conditions]

- Opérateur(s)

[application de prérequis en termes de profils de compétences]

- Modalités et moyens de traitements (ressources)

[application de prérequis en termes de qualité et de viabilité économique]

- Moments et séquences

[application de prérequis en termes de chemin critique]

Le modèle dit quelles activités doivent intervenir, à quel moment, dans quel ordre, sur quoi, pour quels résultats, avec quels moyens, sous quelles conditions. Il dit aussi la même chose pour chaque module de chaque activité et pour chaque opération dans chaque module. Il propose une architecture optimale d'exécution des prestations de traduction sous forme de parcours générique optimal, de parcours particuliers optimaux et de modalités optimales de mise en place d'architectures « opportunistes » correspondant à des combinaisons d'opérateurs suivantes : « traducteur + donneur d'ouvrage », « traducteur + réviseur », « traducteur + donneur d'ouvrage + réviseur », « traducteur + chef de projet », « traducteur + responsable de dossier », et ainsi de suite, sachant que des variantes existent, par exemple, selon que le réviseur se situe côté traducteur ou côté donneur d'ouvrage. Le modèle optimise l'organisation de l'exécution des prestations, garantit la qualité du résultat obtenu en sortie de chaque opération, rationalise la division du travail lorsqu'elle est nécessaire et prévient ses éventuels effets néfastes. Dans la pratique, il combine assurance de qualité (puisque la satisfaction systématique de prérequis interdit l'émergence et la propagation de cônes d'erreur ou de distorsion) et efficacité de gestion. Il est intéressant, à cet égard, de constater que les évolutions récentes des pratiques professionnelles en milieu « industriel » confirment en tout point la validité du modèle général et de ses composantes, à mesure que la pression des délais de mise en marché et la poussée de l'automatisation des tâches obligent à une rationalisation accrue de la mise en œuvre des processus d'exécution des prestations de traduction. Il appelle surtout, au fil des chemins critiques, la mise en œuvre de «modules fonctionnels » 
complexes, imbriqués les uns dans les autres, mais exécutables individuellement aux conditions spécifiées.

\section{APPLICATION PEDAGOGIQUE}

L'application pédagogique reflète en tout point l'organisation du modèle professionnel, puisque l'objectif est de former des professionnels capables d'exécuter les prestations de traduction représentatives du marché réel.

Le cœur de l'application pédagogique est l'architecture d'exécution de prestations de traduction et la formation s'organise par strates ou paliers selon la chronologie des activités et des éléments de prestations en respectant les systèmes de prérequis dégagés par le modèle générique. Les profils de compétences se construisent de manière cohérente et systématique en vertu de leurs contributions attendues à l'exécution de tâches transversales.

Nous avons cessé de faire confiance à un modèle implicite selon lequel, les traducteurs mettant spontanément en œuvre des procédures " généralement » satisfaisantes, il suffit de proposer des correctifs lorsque la démarche spontanée (a) est inopérante ou (b) conduit à un résultat inadéquat. L'application pédagogique du modèle implicite ne serait qu'une série de recettes applicables dans des situations problématiques et la difficulté résiderait uniquement dans l'identification du moment d'incidence et de la cause probable du dysfonctionnement, puisque ce moment et cette cause pourraient se situer en n'importe quel point du parcours forcément aléatoire du traducteur. En pareille hypothèse, il suffirait d'indiquer à tout opérateur concerné l'élément du modèle à mettre en œuvre en lui laissant le soin de vérifier que tous les prérequis (incluant les séquences d'opérations) sont effectivement acquis ${ }^{12}$. On reconnaîtra ici le modèle pédagogique standard en ce sens qu'il est le plus ancien et le plus répandu, sinon le seul ou presque.

En fait, la définition d'objectifs de tâches par strates et donc par faisceaux de compétences selon une rationalité d'achèvement d'éléments de prestation ou de modules ou d'opérations constitue un formidable levier pédagogique ${ }^{13}$ car ceci conduit à fixer les objectifs de formation en termes de compétences, savoir faire, habilités et savoir être et à les organiser en faisceaux cohérents.

Pour construire l'application pédagogique, nous avons décidé qu'il y avait lieu de mettre en place neuf profils de fonctions correspondant à autant de « cœurs de métiers » - ce qui donne en fait treize profils si l'on prend en compte les spécialités de traduction, d'une part, et de localisation, d'autre part. Ces profils sont :

- traduction - traducteur

- généraliste

- spécialisé

- pour les médias

- localisation - localiseur

- de logiciels

- de sites Web

- de jeux vidéo

- relecture-révision (contrôles qualité) - relecteur-réviseur/post-éditeur

- terminologue et phraséologie - terminologue et phraséologue/pré-traducteur

- rédaction - rédacteur

- maîtrise de projets - chef de projets

- webmestrie - webmaster

- ingénierie - ingénieur/logisticien

- gestion des besoins et services linguistiques et de communication - manager linguistique 
Pour construire ces neuf profils de métiers/fonctions, nous avons défini quinze grands domaines de compétence (qui correspondent, sur un autre plan, à autant d'activités). Ces domaines sont, sans ordres de priorités :

- langues

$-\mathrm{A}$

$-\mathrm{B}$

$-\mathrm{C}$

- applications (matières)

- rédaction

- informatique

- outils et ingénieries

- compréhension

- documentation

- terminologie

- phraséologie (inclut mémoires de traductions)

- relecture-révision-réécriture

- comportements

- gestion

- planification et gestion de projets

La colonne vertébrale autour de laquelle s'organisent les domaines de compétence et les faisceaux de compétences est, bien évidemment, la traduction et, plus précisément, la traduction générale, épaulée, en décalage, par la traduction spécialisée puis, de proche en proche, à mesure que les compétences se mettent en place, par la localisation (selon ses trois variantes) et par la traduction pour les médias. Tout le reste du schéma pédagogique vient s'articuler sur la progression en traduction selon le système de prérequis du modèle d'exécution des prestations de traduction.

La progression selon l'axe central de la traduction s'organise selon la logique décrite dans le volume intitulé La qualité en traduction du site www.qualitrad.net. A chacune des phases de la progression correspond une tâche de traduction cohérente au sens où elle aboutit à la production en tout ou partie d'une forme de traduction économiquement et professionnellement justifiée. L'enchaînement est de type (1) traduction signalétique, (2) traductions synoptiques, (3) traduction aménagée (a) à priorité documentaire et (b) banalisée puis (4) traduction absolue - étant entendu que, dans chaque cas, la traduction sélective précède la traduction intégrale ${ }^{14}$ et que les niveaux de finition sont eux-mêmes étagés du brut au livrable puis au diffusable.

L'architecture de formation se construit donc selon une succession de vingt-quatre paliers cohérents, dont la concaténation aboutit à la mise en place de l'ensemble des profils de compétences requis pour la bonne exécution de prestations de traduction. On retrouve ces 24 paliers sur chacun des axes de domaines précédemment définis. Le passage d'un niveau à l'autre intervient quand l'étudiant maitrise la totalité des prérequis et ne peut pas intervenir tant que ces prérequis font défaut $^{15}$. Il est également important de préciser que l'incidence de la traduction est retardée : elle n'intervient qu'à l'étape 8 de la série des 24 étapes, parce qu'aucune forme de traduction n'est envisagée tant que tous les prérequis ne sont pas en place.

Pour chacun des domaines à l'exception des langues, la formation s'organise en X. Le X se compose d'un $\mathrm{V}$ initial auquel vient s'ajouter un $\Lambda$. Le V concerne tout élément de formation nécessaire, en tant que prérequis, à l'exécution de la forme de traduction considérée. Le $\Lambda$ marque l'ouverture vers le métier correspondant au domaine considéré, au-delà des compétences de domaines requises pour la traduction proprement dite. 
Ainsi, par exemple, pour le domaine « terminologie », le modèle met en place, dans un premier temps, des enseignements et pratiques de terminographie traductive considérée exclusivement sous l'angle de ses préréquisitions dans une perspective d'exécution de traductions : chaque élément de compétence terminographique contribuant à la bonne exécution des traductions est mis en place avant que l'exécution de la tâche de traduction le requière. Un peu plus tard, mais toujours dans la même optique de traduction, la terminographie s'élargit à la préparation et à l'exploitation des ressources plus générales dites « terminologies pour traducteurs ». Tout est, dans ces phases pré-traductives, focalisé sur la traduction (d'où la représentation en V).

Puis, une fois toutes les compétences terminographiques traductives acquises et validées, le V s'inverse et la terminographie-terminologie s'ouvre au-delà de la traduction. Il s'agit alors de mettre en place des compétences de terminographie orientées vers des tâches autres que la traduction [rédaction, formation, aménagement, etc.] puis d'ouvrir sur la terminologie (discipline ou science) et l'ontologie. On passe ainsi, progressivement, d'un profil de compétences de terminographe-terminologue exigibles dans les limites de l'exécution de prestations de traduction à un profil de métier de terminographe-terminologue. Et, dans la variante la plus poussée du modèle (niveau master 2), le domaine « terminologie » s'ouvre également, au final, sur des activités de recherche et développement ainsi que sur des activités de gestion des problématiques terminologiques et terminographiques de la communication multilingue et multimédia. L'annexe 3 présente le schéma global du domaine « terminologie ».

On retrouve ainsi, sur l'axe de chacun des domaines, une progression en quatre grands volets :

1) Mobilisation des compétences requises pour l'exécution de prestations de traduction [compétences de tâche]

2) Ajout de compétences de même domaine fondant un « vrai » profil professionnel dans la spécialité [compétences de métier]

3) Etude de la discipline/des disciplines ou de la science concernée

4) Recherche et développement

Le modèle d'exécution de prestations de traduction fonde la construction du volet initial de l'architecture de formation dans chaque domaine. Il remplit à cet égard une double fonction. Tout d'abord, il dit quelles sont les compétences à mettre en place et dans quel ordre. Ensuite, et ce n'est pas son moindre mérite, il écarte provisoirement ce qui relève davantage d'un profil de compétences de métier autre que celui du traducteur. Il permet ainsi, en fixant des priorités, un meilleur ciblage des objectifs pédagogiques et une meilleure exploitation des ressources pédagogiques.

Le modèle a également généré un principe pédagogique absolu : quelle que soit la tâche assignée à quelque moment que ce soit des parcours de formation, elle s'exécute en configuration de gestion de projets. Ceci oblige à définir un chemin critique, à mesurer les entrées et sorties de processus, et à mettre en œuvre des opérations ou modules ou éléments de prestation dont les tenants et les aboutissants ont été parfaitement identifiés et optimisés. Le principe de la gestion de projets s'applique à toutes les activités des étudiants du CFTTR, sans la moindre exception. Les avantages que l'on en retire sur tous les plans sont incommensurables ${ }^{16}$.

Enfin, le modèle a permis de développer ${ }^{17}$ une ingénierie pédagogique complète. Quelle que soit la tâche, l'élément de prestation, l'activité, l'opération ou le groupe d'opérations à exécuter, le dispositif d'appui comporte :

1) un référentiel complet en ligne [site Web]

Ce référentiel présente ou indique tous les éléments de savoirs, savoir faire, savoir être et stratégies nécessaires. 
Dans les périodes d'exécution de projets, le référentiel en ligne inclut un référent en ligne - le formateur - qui répond à toute question posée dans le délai le plus réduit [pas plus d'une dizaine de minutes].

2) un cahier des charges générique [fichier disponible en permanence]

Ce cahier des charges fixe : (a) l'objet - avec prérequis, (b) les objectifs, (c) les ressources à mettre en œuvre, (d) les enjeux, (e) les risques, (f) les stratégies ou procédures à mettre en œuvre pour l'exécution de toute prestation relevant de la catégorie considérée.

3) un « kit » de travail [fichiers disponibles en permanence]

Ce kit est un développement logiciel (formulaire) qui oblige à consigner effectivement tous les éléments critiques pour chacune des opérations effectuées : objet considéré, filtres, contraintes, éléments liés, résultat du traitement, validation, etc.

Le kit est accompagné de trois instruments : (a) son descriptif, (b) son mode d'emploi, et (c) le cahier des charges de sa mise en ouvre.

L'ensemble « kit + documents d'accompagnement » est développé chaque année par les étudiants eux-mêmes selon les prescriptions du formateur, au titre des activités de recherche et développement. La réalisation de cet ensemble constitue le meilleur des apprentissages des prescriptions applicables et du modèle dans sa totalité.

4) Une procédure ou un jeu de procédures [fichiers disponibles en permanence]

Les procédures concernent les opérations. Elles sont éventuellement regroupées en jeux applicables à des modules. Elles renvoient, selon les exigences particulières, aux divers éléments de kit et à leurs exploitations.

Un cahier des charges particulier fixe, dans chaque cas, les conditions particulières applicables en sus de celles spécifiées dans le cahier des charges générique. Ce cahier des charges particulier insiste, en contexte pédagogique, sur les procédures d'exécution des opérations.

\section{CONCLUSION}

L'exécution des prestations de traduction se prête à une modélisation en chemin critique par combinaison d'opérations élémentaires définissant des modules fonctionnels, puis des activités, puis des éléments de prestation. Chaque opération s'analyse en termes d'objet, objectif, enjeux, risques, stratégies et procédures. Chaque opération impose des prérequis et son exécution appelle la mise en œuvre de faisceaux de compétences clairement identifiées.

On peut ainsi construire des organisations de travail optimales en contexte professionnel en s'appuyant sur des profils de compétences par fonctions. Ces profils de compétences définissent à leur tour des objectifs de formation systématiques par strates. Ces objectifs justifient la mise en place d'architectures de formation organisées selon le système d'emboîtement de prérequis du modèle professionnel et mobilisant les divers domaines de compétence relevés. Et, pour favoriser la maîtrise des compétences requises, l'application pédagogique repose sur un dispositif de formation extrêmement contraignant au cœur duquel se trouvent les procédures d'exécution des diverses opérations et combinaisons d'opérations.

\section{NOTES}

1. Il n'était pas alors question de «prestation de traduction » puisque nous n'étions concerné que par la traduction de la composante textuelle

2. Ainsi a débuté au CFTTR la mise en place d'un dispositif qui, sans porter le nom de « dispositif d'assurance qualité », en présentait les caractéristiques essentielles. L'intérêt majeur de la transposition résidait dans l'allègement de la charge supportée par le formateur dans un contexte de sous-encadrement chronique. 
3. Cette seconde analyse est renouvelée chaque semestre depuis 1986. Elle s'effectue par analyse des descriptifs d'activités contenus dans les rapports de stages et consultation systématique des personnes et organismes membres du conseil de perfectionnement du CFTTR.

4. Matériau/instrument dont les variables sont celles évoquées précédemment.

5. La traduction étant avant tout une industrie de transformation.

6. Comme cet exemple le montre, l'activité peut également être élément de prestation.

7. Tâches achevées selon les prévisions pour que le résultat de l'opération/du module/de l'activité soit disponible à la date prévue ou promise.

8. Modélisation du processus d'exécution des traductions, META Vol. 50 n², pp. 643sq

9. Exemple d'une opération de transformation telle que représentée à l'annexe 1.

10. Les spécifications peuvent être génériques ou spécifiques, normatives ou « locales », éparses ou organisées en cahier des charges, diffuses ou organisées en procédure.

11. Les contenus de prestations se diversifient à tel point qu'il devient impossible de parler d'un profil de compétences du traducteur sauf à énumérer les fonctions que celui-ci devra assurer et à construire son profil sur la base de la somme des compétences requises pour et par les fonctions identifiées (nécessaires, requises, souhaitées).

12. Ou en espérant qu'ils le sont et c'est bien là que se situe le problème car l'analyse montre que toute défaillance de processus est provoquée - directement ou indirectement - par l'absence ou la carence d'un prérequis.

13. On notera avec intérêt que c'est l'émulation professionnelle sous-tendant la pédagogie par projets du centre de formation de traducteurs qui a permis l'optimisation finale du modèle avant qu'il pénètre les milieux professionnels. 14. Voir www.qualitrad.net, volumes 1 et 2.

15. Ce qui signifie, entre autres, que les temps de formation par phase ne peuvent pas être déterminées à l'avance. On peut ainsi dire que le temps moyen de maîtrise totale de la traduction synoptique est en gros, d'un semestre à raison d'une heure de cours et de trois heures d'application par semaine mais ceci signifie qu'il faudra douze semaines pour certains étudiants et trois pour d'autres.

16. Voir, du même auteur, Pédagogie par projets in Formation des traducteurs (2) Actes du colloque international sur la traduction (Rennes 2004) [Version PDF téléchargeable à www.colloque.net]

17. On dirait, plus justement, qu'il n'aurait pu se mettre en place sans ce dispositif d'appui. 


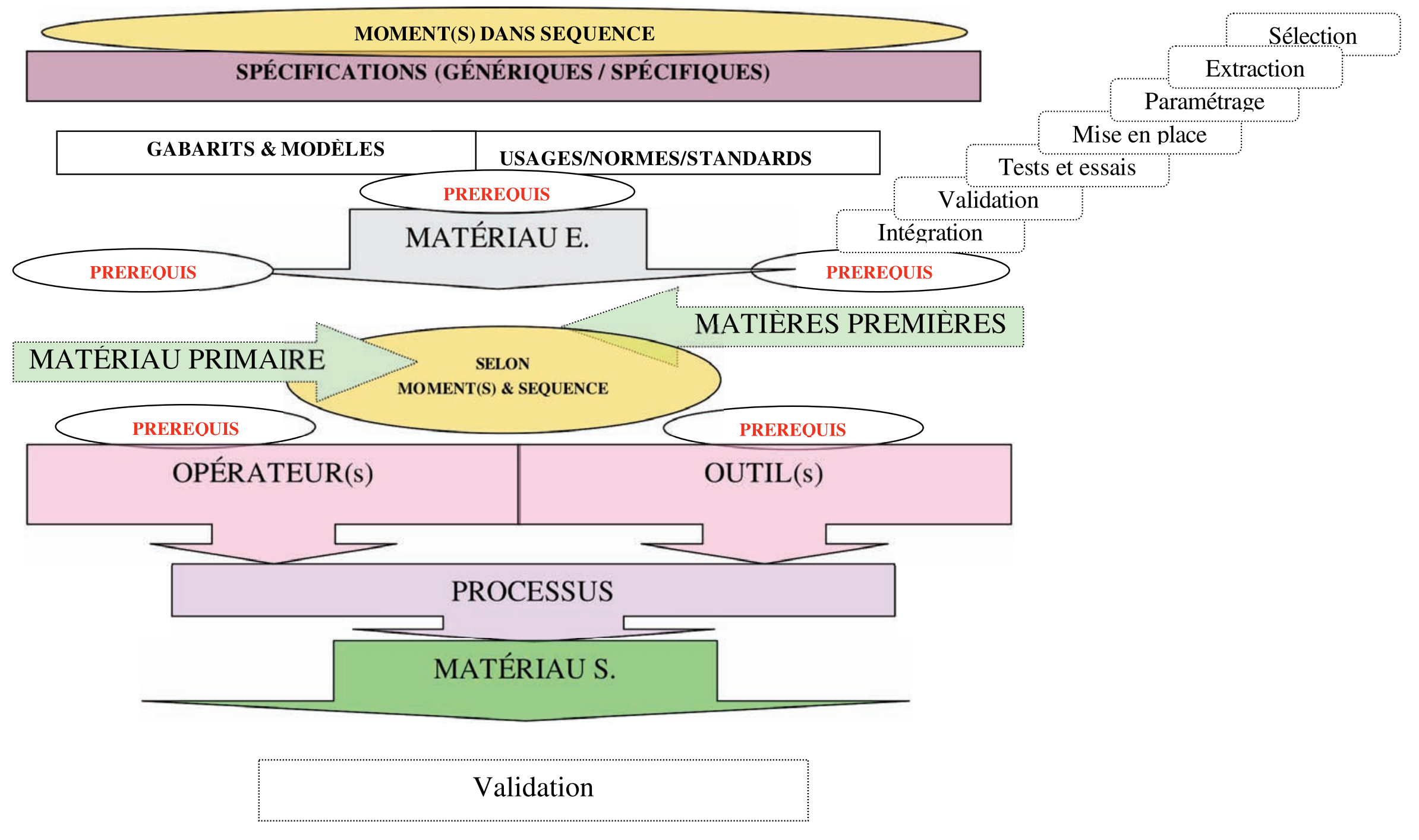

Annexe 1 : Activité de transformation 


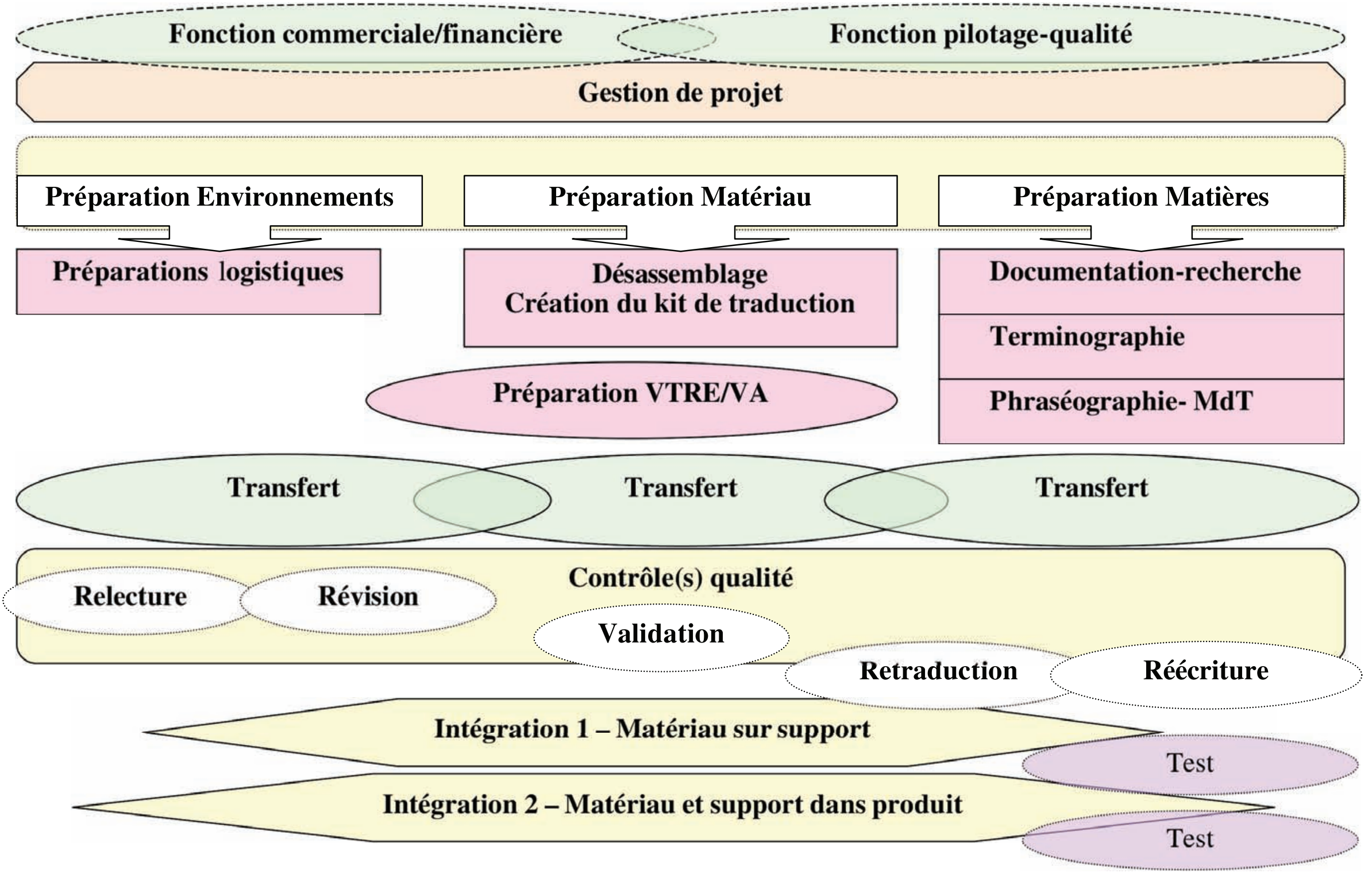

Annexe 2 : Fonctions d'exécution de prestations de traduction (côté traducteur) 


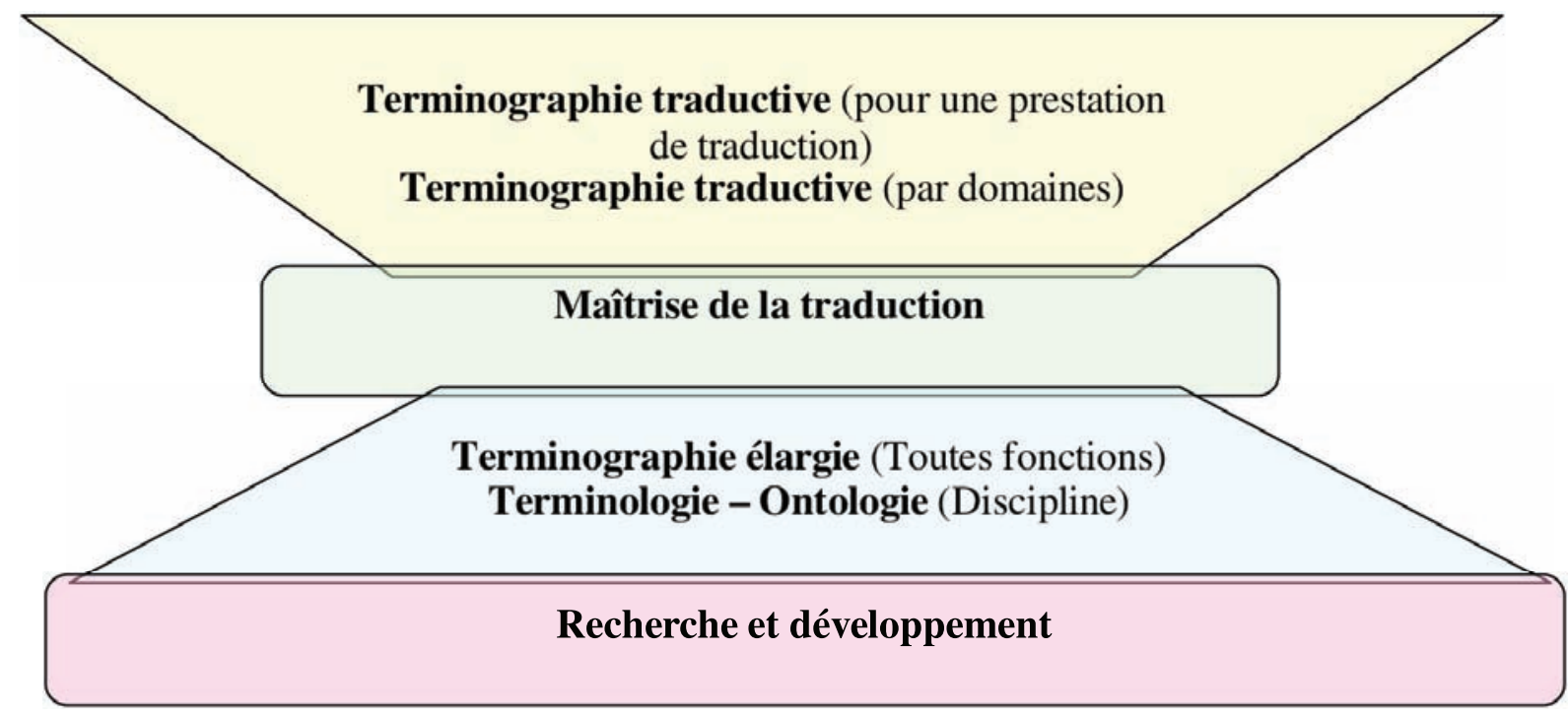

Annexe 3 : Schéma global du domaine 'terminologie'.

Le détail de l'architecture de formation sera disponible en 2005 à www.translator-training.org 